\title{
ON SYSTEMS OF CLAIRAUT TYPE
}

\author{
By ShyUichi IZUMIYA and Yasuhiro KurokaWA
}

\section{Introduction}

About 260 years ago Alex Claude Clairaut [1] studied the following equation which is called the Clairaut equation now : $y=x \cdot \frac{d y}{d x}+f\left(\frac{d y}{d x}\right)$. It is usually taught in the first or second year course of calculus in the university and treated as one of the typical examples of non-linear equations that are easily solved. Moreover it has a quite beautiful geometric structure as follows : There exists a "general solution" that consists of lines ; $y=t \cdot x+f(t)$, where $t$ is a parameter and the singular solution is the envelope of such a family (Fig. 1).

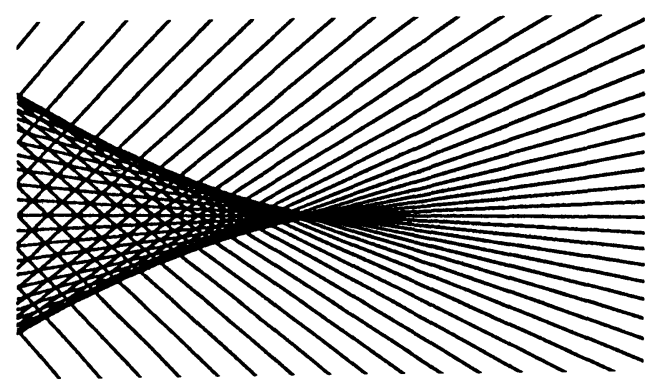

Fig. 1

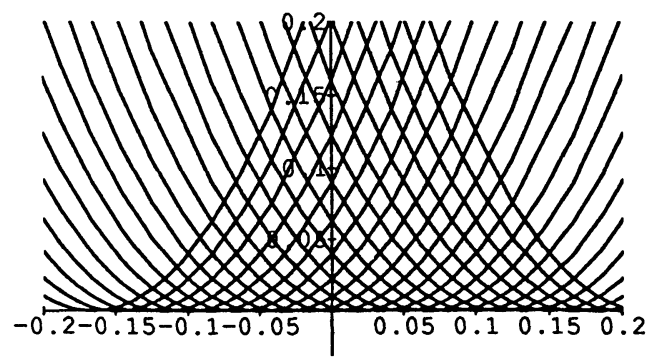

Fig. 2

In this article we consider equations with the same geometric structure as the Cläiraut equation. Here we give another example as follows : $y-\left(\frac{d y}{d x}\right)^{2}=0$. We can easily solve this equation: the "general solution" is given by $y=\frac{1}{4}(x+t)^{2}$, where $t$ is a parameter. Here, the "singular solution" is given by $y=0$ that is the envelope of the family of graphs of the "general solution". The "general solution" of this equation does not consist of lines. However, the "singular solution" is the envelope of the graphs of the "general solution" like as the Clairaut equation (Fig. 2).

We will refer such an equation as a Clairaut-type equation. Our purpose in this note is to give a characterization of Clairau-type equations and to classify these equation under a "nice" equaivalence relation.

In this note we will sketch our discussions without proofs. The detailed paper will be published elswhere soon.

All maps considered here are differentiable of class $C^{\infty}$, unless stated otherwise. 


\section{Framework}

In this section we give the basic framework to study systems of Clairaut types. A system of first order differential equation is most naturally interpreted as being a closed subset of the 1-jet space of functions of $n$-variables $J^{1}\left(\mathbf{R}^{n}, \mathbf{R}\right)$. Unless the contrary is specifically stated, we use the following definition. A system of first order differential equation (or briefly, an equation) is a submanifold germ $\left(E, z_{0}\right) \subset\left(J^{1}\left(\mathbf{R}^{n}, \mathbf{R}\right), z_{0}\right)$. Let $\theta$ be the canonical contact form on $J^{1}\left(\mathbf{R}^{n}, \mathbf{R}\right)$ which is given by $\theta=d y-\sum_{t=1}^{n} p_{i} d x_{i}$, where $(x, y, p)$ are canonical coordinates of $J^{1}\left(\mathbf{R}^{n}, \mathbf{R}\right)$. Let $\pi: J^{1}\left(\mathbf{R}^{n}, \mathbf{R}\right) \rightarrow \mathbf{R}^{n} \times \mathbf{R}$ be the canonical projection. We say that $z_{0}$ is a $\pi$-singular point if rank $d(\pi \mid E)_{z_{0}}<n+1$. We denote the set of $\pi$-singular points by $\Sigma_{\pi}(E)$ and $\pi\left(\Sigma_{\pi}(E)\right)=D_{E}$. We call the set $D_{E}$ the discriminant set of the equation $\left(E, z_{0}\right)$. Since we only consider the local properties, we adopt the following two kinds of representations:

An equation germ is

(1) a submersion germ $F:\left(J^{1}\left(\mathbf{R}^{n}, \mathbf{R}\right), z_{0}\right) \rightarrow\left(\mathbf{R}^{d}, 0\right)$

or

(2) an immersion germ $f:\left(\mathbf{R}^{r}, 0\right) \rightarrow\left(J^{1}\left(\mathbf{R}^{n}, \mathbf{R}\right), z_{0}\right)$, where $r=2 n+1-d(1 \leq d \leq n)$ and $\left(E, z_{0}\right)=\left(F^{-1}(0), z_{0}\right)=\left(\right.$ Image $\left.f, z_{0}\right)$.

We use either notations depend on the situations. We define a geometric solution of $\left(E, z_{0}\right)$ to be an $n$-dimensional manifold $i:\left(L, z_{0}\right) \subset\left(J^{1}\left(\mathbf{R}^{n}, \mathbf{R}\right), z_{0}\right)$ such that $\theta \mid L=0$ and $L \subset\left(E, z_{0}\right)$ (i.e. a Legendrian submanifold which is contained in $\left(E, z_{0}\right)$ ). Let $g: \mathbf{R}^{n} \rightarrow \mathbf{R}$ be a smooth function. Then the jet extension $j^{1} g: \mathbf{R}^{n} \rightarrow J^{1}\left(\mathbf{R}^{n}, \mathbf{R}\right)$ is a Legendrian embedding. Hence, in our terminology, the classical solution of $\left(E, z_{0}\right)$ is a smooth function germ $g$ such that $\left(j^{1} g\left(\mathbf{R}^{n}\right), z_{0}\right) \subset\left(E, z_{0}\right)$. On the other hand, we can show that a geometric solution $i$ is given by (at least locally) a jet extension of a smooth function if and only if $\pi \circ i$ is a non-singular map. We say that $z_{0} \in L$ is a Legendrian singular point if $z_{0}$ is a singular point of $\pi \circ i$. An equation $\left(E, z_{0}\right)$ is said to be completely integrable if there exists a foliation by geometric solutions on $\left(E, z_{0}\right)$. In this case such a foliation is called a complete solution of $\left(E, z_{0}\right)$. We say that a complete solution is classical if each leaf of the complete solution is classical (i.e., Legendrian non-singular).

\section{Characterization theorems}

In this section we give characterizations of equations with classical complete solution. We adopt the representation by submersions: an equation is a submersion germ $F$ : $\left(J^{1}\left(\mathbf{R}^{n}, \mathbf{R}\right), z_{0}\right) \rightarrow\left(\mathbf{R}^{d}, 0\right)$, where $1 \leq d \leq n$.

An equation $F=0$ is said to be Clairaut type if there exist smooth function germs $B_{\jmath \imath}, A_{i k}^{\ell}:\left(J^{1}\left(\mathbf{R}^{n}, \mathbf{R}, z_{0}\right) \rightarrow \mathbf{R}\right.$ for $i, j=1, \ldots, n, k=1, \ldots, d$ and $\ell=1, \ldots, d$ such that

$$
\frac{\partial F_{\ell}}{\partial x_{i}}+p_{i} \frac{\partial F_{\ell}}{\partial y}=\sum_{\jmath=1}^{n} B_{\jmath} \frac{\partial F_{\ell}}{\partial p_{j}}+\sum_{k=1}^{d} A_{i k}^{\ell} F_{k} \quad(i=1, \ldots, n \text { and } \ell=1, \ldots, d)
$$

and satisfy that

$$
\begin{aligned}
B_{j i} & =B_{i \jmath} \\
\frac{\partial B_{j k}}{\partial x_{i}}+p_{i} \frac{\partial B_{j k}}{\partial y}+\sum_{\ell=1}^{n} B_{\ell i} \frac{\partial B_{j k}}{\partial p_{\ell}} & =\frac{\partial B_{\jmath \imath}}{\partial x_{k}}+p_{k} \frac{\partial B_{\jmath \ell}}{\partial y}+\sum_{\ell=1}^{n} B_{\ell k} \frac{\partial B_{j i}}{\partial p_{\ell}}
\end{aligned}
$$


at any $z \in\left(F^{-1}(0), z_{0}\right)$ for $i, j, k=1, \ldots, n$.

We have the following characterization theorem.

THEOREM 2.1. For an equation germ $F=0$, the following are equivalent.

(1) $F=0$ is the Clarraut type equation.

(2) $F=0$ has a (classical) complete solution.

In this case, if $\Sigma_{\pi}\left(F^{-1}(0)\right) \neq \emptyset$, then $\Sigma_{\pi}\left(F^{-1}(0)\right)$ is a geometric solution (i.e. the singular solution) of $F=0$ and the discriminant set $D_{F^{-1}(0)}$ is the envelope of the family of graphs of the complete solution.

We now give two examples which describe the above assertion.

Examples 2.2. 1) The following equation is a generalization of the classical Clairaut equation:

$$
F_{i}\left(p_{1}, \ldots, p_{n}\right)=0 \quad(i=1, \ldots, d-1), F_{d}(x, y, p)=y-\sum_{i=1}^{n} p_{i} x_{i}-f\left(p_{1}, \ldots, p_{n}\right)=0
$$

where $F_{\imath}, f$ are function germ.

Since $F=\left(F_{1}, \ldots, F_{d}\right)$ is a submersion, we have $\operatorname{rank}\left(\frac{\partial F_{2}}{\partial x_{j}}\right)=d-1$ Then the set $F^{-1}(0)$ is locally parametrized by an immersion $a(t)=\left(a_{1}(t), \ldots, a_{n}(t)\right)$, where $t=\left(t_{1}, \ldots, t_{n-d+1}\right)$. It follows that we can get a classical complete solution

$$
y=\sum_{i=1}^{n} a_{i}(t) x_{i}+f\left(a_{1}(t), \ldots, a_{n}(t)\right)
$$

We can easily check that

$$
\frac{\partial F_{\ell}}{\partial x_{i}}+p_{i} \frac{\partial F_{\ell}}{\partial y}=0
$$

on $F^{-1}(0)$. This means that we can choose $B_{i j}=0$.

2) Consider the following equation : $F_{1}=p_{1}^{2}-y=0, F_{2}=p_{2}=0(n=2)$.

Then we have

$$
\begin{aligned}
& \frac{\partial F_{1}}{\partial x_{1}}+p_{1} \frac{\partial F_{1}}{\partial y}=-p_{1}, \frac{\partial F_{1}}{\partial x_{2}}+p_{2} \frac{\partial F_{1}}{\partial y}=-p_{2} \\
& \frac{\partial F_{2}}{\partial x_{1}}+p_{1} \frac{\partial F_{2}}{\partial y}=0, \frac{\partial F_{2}}{\partial x_{2}}+p_{2} \frac{\partial F_{2}}{\partial y}=0
\end{aligned}
$$

and

It follows that

$$
\frac{\partial F_{1}}{\partial p_{1}}=2 p_{1}, \frac{\partial F_{1}}{\partial p_{2}}=0, \frac{\partial F_{2}}{\partial p_{1}}=0, \frac{\partial F_{2}}{\partial p_{2}}=1
$$

$$
\begin{aligned}
& \frac{\partial F_{1}}{\partial x_{1}}+p_{1} \frac{\partial F_{1}}{\partial y}=-\frac{1}{2} \cdot \frac{\partial F_{1}}{\partial p_{1}}+0 \cdot \frac{\partial F_{1}}{\partial p_{2}}+0 \cdot F_{1}+0 \cdot F_{2} \\
& \frac{\partial F_{1}}{\partial x_{2}}+p_{2} \frac{\partial F_{1}}{\partial y}=0 \cdot \frac{\partial F_{1}}{\partial p_{1}}+0 \cdot \frac{\partial F_{1}}{\partial p_{2}}+0 \cdot F_{1}-1 \cdot F_{2} \\
& \frac{\partial F_{2}}{\partial x_{1}}+p_{1} \frac{\partial F_{2}}{\partial y}=-\frac{1}{2} \cdot \frac{\partial F_{2}}{\partial p_{1}}+0 \cdot \frac{\partial F_{2}}{\partial p_{1}}+0 \cdot F_{1}+0 \cdot F_{2}
\end{aligned}
$$




$$
\frac{\partial F_{2}}{\partial x_{2}}+p_{2} \frac{\partial F_{2}}{\partial y}=0 \cdot \frac{\partial F_{2}}{\partial p_{1}}+0 \cdot \frac{\partial F_{2}}{\partial p_{2}}+0 \cdot F_{1}+0 \cdot F_{2} .
$$

The complete solution is given by $y=\frac{1}{4}\left(x_{1}+t\right)^{2}$.

We can also characterize equations with (classical) complete solutions that consist of hyperplanes.

THEOREM 2.3. For an equation $F:\left(J^{1}\left(\mathbf{R}^{n}, \mathbf{R}\right), z_{0}\right) \rightarrow\left(\mathbf{R}^{d}, 0\right)$ with $\Sigma_{\pi}\left(F^{-1}(0)\right) \neq$ $\emptyset$, the following are equivalent.

(1) There exıst smooth function germs $A_{i k}^{\ell}:\left(J^{1}\left(\mathbf{R}^{n}, \mathbf{R}\right), z_{0}\right) \rightarrow \mathbf{R}$ such that

$$
\frac{\partial F_{\ell}}{\partial x_{i}}+p_{i} \frac{\partial F_{\ell}}{\partial y}=\sum_{k=1}^{d} A_{i k}^{\ell} F_{k} \quad \text { for } i=1, \ldots, n, \ell=1, \ldots, d .
$$

(2) There exists a classical complete solution of $F=0$ such that all members are hyperplanes.

(3) There exists a submersion germ $G:\left(\mathbf{R}^{n}, p_{0}\right) \rightarrow\left(\mathbf{R}^{d}, 0\right)$ and a function germ $f:\left(\mathbf{R}^{n}, p_{0}\right) \rightarrow \mathbf{R}$ such that

$$
F^{-1}(0)=\left\{(x, y, p) \mid G\left(p_{1}, \ldots, p_{n}\right)=0 \text { and } y=\sum_{i=1}^{n} x_{i} p_{i}-f\left(p_{1}, \ldots, p_{n}\right)\right\}
$$

We call the equation in the above theorem a Clairaut system.

\section{Holonomic systems of Clairaut type}

In this section we consider the classification problem among systems of Clairaut type under the equivalence relation induced by the group of point transformations in the sense of Sophus Lie. We stick to holonomic systems and adopt the following representation. A holonomic system of first order differential equation germ (or, briefly, a holonomic system) is defined to be an immersion germ $f:\left(\mathbf{R}^{n+1}, 0\right) \rightarrow J^{1}\left(\mathbf{R}^{n}, \mathbf{R}\right)$. We introduce an equivalence relation among systems under the group of point transformations of $\mathbf{R}^{n} \times \mathbf{R}$. A point transformation $\phi$ on $\mathbf{R}^{n} \times \mathbf{R}$ is, by definition, a diffeomorphism of $\mathbf{R}^{n} \times \mathbf{R}$ onto itself.

To define a lift of $\phi$, we give a contact manifold which is a fiberwise compactification of $J^{1}\left(\mathbf{R}^{n}, \mathbf{R}\right)$. Let $\tilde{\pi}: P T^{*}\left(\mathbf{R}^{n} \times \mathbf{R}\right) \rightarrow \mathbf{R}^{n} \times \mathbf{R}$ be a projective cotangent bundle over $\mathbf{R}^{n} \times \mathbf{R}$ which contains $\pi: J^{1}\left(\mathbf{R}^{n}, \mathbf{R}\right) \rightarrow \mathbf{R}^{n} \times \mathbf{R}$ as an affine part. Then we have a canonical contact lift $\hat{\phi}: P T^{*}\left(\mathbf{R}^{n} \times \mathbf{R}\right) \rightarrow P T^{*}\left(\mathbf{R}^{n} \times \mathbf{R}\right)$ of $\phi$. Let $f, g:\left(\mathbf{R}^{n+1}, 0\right) \rightarrow J^{1}\left(\mathbf{R}^{n}, \mathbf{R}\right)$ be equation germs. Following Lie, we say that $f$ and $g$ are equivalent as equatıons if there exist a diffeomorphism germ $\psi:\left(\mathbf{R}^{n+1}, 0\right) \rightarrow\left(\mathbf{R}^{n+1}, 0\right)$ and a point transformation $\phi:\left(\mathbf{R}^{n} \times \mathbf{R}, \pi\left(z_{0}\right)\right) \rightarrow\left(\mathbf{R}^{n} \times \mathbf{R}, \pi\left(z_{1}\right)\right)$ such that the lift $\hat{\phi}$ of $\phi$ satisfies that $\hat{\phi}\left(z_{0}\right)=z_{1}$ and $\hat{\phi} \circ f=g \circ \psi$, where $z_{0}=f(0)$ and $z_{1}=g(0)$. By the definition of complete integrability and Frobenius' theorem we may define as follows. We say that $f$ is completely integrable if there exists a submersion germ $\mu:\left(\mathbf{R}^{n+1}, 0\right) \rightarrow \mathbf{R}$ such that $\langle d \mu\rangle_{\mathcal{E}_{u}} \supset\left\langle f^{*} \theta\right\rangle_{\mathcal{E}_{u}}$, where $\mathcal{E}_{u}$ is the ring of smooth function germs on $\left(\mathbf{R}^{n+1}, 0\right)$ and its unique maximal ideal is denoted by $\mathfrak{M}_{u}$. Here, $u=\left(u_{1}, \ldots, u_{n+1}\right)$ are canonical coordinates of $\left(\mathbf{R}^{n+1}, 0\right)$. We call $\mu$ a complete integral of $f$ and the pair $(\mu, f):\left(\mathbf{R}^{n+1}, 0\right) \rightarrow \mathbf{R} \times J^{1}\left(\mathbf{R}^{n}, \mathbf{R}\right)$ is called a holonomic system with complete integral. Here, we can observe that $\pi \circ f\left(\mu^{-1}(t)\right)$ 
is the graph of the solution in $\mathbf{R}^{n} \times \mathbf{R}$. If $\pi \circ f \mid \mu^{-1}(t)$ are non-singular map germs for any $t \in(\mathbf{R}, \mu(0))$, then $\left\{\pi \circ f\left(\mu^{-1}(t)\right)\right\}_{t \in \mathbf{R}}$ is the family of graphs of a classical complete solution (cf. $\S 2$ ). We call such a system a holonomıc system of Claıraut type. These situation lead us to the following definition. Let $(\mu, g)$ be a pair of a map germ $g:\left(\mathbf{R}^{n+1}, 0\right) \rightarrow\left(\mathbf{R}^{n} \times \mathbf{R}, 0\right)$ and a submersion germ $\mu:\left(\mathbf{R}^{n+1}, 0\right) \rightarrow(\mathbf{R}, 0)$. Then the diagram

$$
(\mathbf{R}, 0) \stackrel{\mu}{\longleftarrow}\left(\mathbf{R}^{n+1}, 0\right) \stackrel{g}{\longrightarrow}\left(\mathbf{R}^{n} \times \mathbf{R}, 0\right)
$$

or briefly $(\mu, g)$, is called a (holonomıc) integral diagram if there exists a holonomic system $f:\left(\mathbf{R}^{n+1}, 0\right) \rightarrow J^{1}\left(\mathbf{R}^{n}, \mathbf{R}\right)$ such that $(\mu, f)$ is an equation germ with complete integral and $\pi \circ f=g$, and we say that the integral diagram $(\mu, g)$ is induced by $f$. If $f$ is a system of Clairaut type, then $(\mu, \pi \circ f)$ is called of Clairaut type. Furthermore we introduce an equivalence relation among integral diagrams. Let $(\mu, g)$ and $\left(\mu^{\prime}, g^{\prime}\right)$ be integral diagrams. Then $(\mu, g)$ and $\left(\mu^{\prime}, g^{\prime}\right)$ are equıvalent (respectıvely, strictly equıvalent) if the diagram

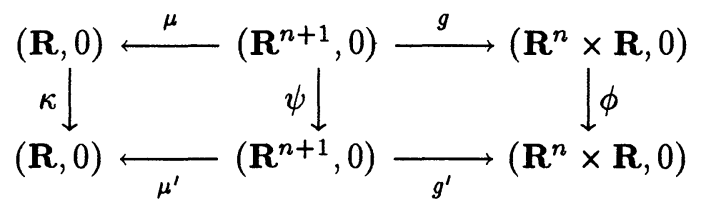

commutes for some diffeomorphism germs $\kappa, \psi$ and $\phi$ (respectively, $\kappa=i d_{\mathbf{R}}$ ).

In [3] it has been shown the following theorem.

Theorem 3.1. Let $(\mu, f)$ and $\left(\mu^{\prime}, f^{\prime}\right):\left(\mathbf{R}^{n+1}, 0\right) \rightarrow\left(\mathbf{R} \times J^{1}\left(\mathbf{R}^{n}, \mathbf{R}\right), 0 \times v\right)$ be holonomic system with complete integral such that the set of critical points of $\pi \circ f$ and $\pi \circ f^{\prime}$ are closed sets without interior points. Then the followings are equivalent:

(1) $f$ and $f^{\prime}$ are equivalent as equations.

(2) $(\mu, \pi \circ f)$ and $\left(\mu^{\prime}, \pi \circ f^{\prime}\right)$ are equivalent as integral diagrams.

Moreover, it has been given generic classifications of holonomic integral diagrams by the strict equivalence in the case when $1 \leq n \leq 3$. For general $n$, by the technical reason it is very hard to give classification. However, we can give a generic classification of holonomic systems of Clairaut type. We describe the meaning of genericity in the sequel. We briefly review the theory of one-parameter Legendrian unfoldings in $[3,4]$. We now consider the 1-jet bundle $J^{1}\left(\mathbf{R} \times \mathbf{R}^{n}, \mathbf{R}\right)$ and the canonical 1-form $\Theta$ on the space. Let $\left(t, x_{1}, \ldots, x_{n}\right)$ be the canonical coordinate on $\mathbf{R} \times \mathbf{R}^{n}$ and $\left(t, x_{1}, \ldots, x_{n}, y, q, p_{1}, \ldots, p_{n}\right)$ be the corresponding coordinate on $J^{1}\left(\mathbf{R} \times \mathbf{R}^{n}, \mathbf{R}\right)$. Then the canonical 1-form is given by $\Theta=d y-\sum_{\imath=1}^{n} p_{i} d x_{i}-q d t=\theta-q d t$. We also have the natural projection

$$
\Pi: J^{1}\left(\mathbf{R} \times \mathbf{R}^{n}, \mathbf{R}\right) \rightarrow\left(\mathbf{R} \times \mathbf{R}^{n}\right) \times \mathbf{R}
$$

defined by $\Pi(t, x, y, q, p)=(t, x, y)$. We call the above 1-jet bundle an unfolded 1-jet bundle. Let $(\mu, f)$ be a holonomic system with complete integral. Then there exists a unique element $h \in \mathcal{E}_{r}$ such that $\ell^{*} \theta=h \cdot d \mu$, where $\mathcal{E}_{r}$ is the ring of function germs of $r$-variables at the origin. Define a map germ

$$
\ell_{(\mu, f)}:\left(R, u_{0}\right) \rightarrow J^{1}\left(\mathbf{R} \times \mathbf{R}^{n}, \mathbf{R}\right)
$$


by

$$
\ell_{(\mu, f)}(u)=(\mu(u), x \circ \ell(u), y \circ \ell(u), h(u), p \circ \ell(u)) .
$$

Then we can easily show that $\ell_{(\mu, f)}$ is a Legendrian immersion germ. We call $\ell_{(\mu, f)} a$ complete Legendrian unfolding associated wrth $(\mu, f)$. By the aid of the notion of Legendrian unfoldings, holonomic systems of Clairaut type are characterized as follows :

Proposition 3.2. Let $(\mu, f):\left(\mathbf{R}^{n+1}, 0\right) \rightarrow \mathbf{R} \times J^{1}\left(\mathbf{R}^{n}, \mathbf{R}\right)$ be a holonomic system with complete integral. Then $(\mu, f)$ is a holonomic system of Clairaut type if and only if $\ell_{(\mu, f)}$ is Legendrian non-singular.

A complete Legendrian unfolding $\ell_{(\mu, f)}$ associated with $(\mu, f)$ is called a Legendrian unfolding of Claıraut type if $\ell_{(\mu, f)}$ is a holonomic system of Clairaut type.

Returning to the study of equations with complete integral, we now establish the notion of the genericity.

Let $U \subset \mathbf{R}^{n+1}$ be an open set. We denote by $\operatorname{Int}\left(U, \mathbf{R} \times J^{1}\left(\mathbf{R}^{n}, \mathbf{R}\right)\right)$ the set of systems with complete integral $(\mu, f): U \rightarrow \mathbf{R} \times J^{1}\left(\mathbf{R}^{n}, \mathbf{R}\right)$. We also define $L\left(U, J^{1}\left(\mathbf{R} \times \mathbf{R}^{n}, \mathbf{R}\right)\right)$ to be the set of complete Legendrian unfoldings $\ell_{(\mu, f)}: U \rightarrow J^{1}\left(\mathbf{R} \times \mathbf{R}^{n}, \mathbf{R}\right)$.

These sets are topological spaces equipped with the Whitney $C^{\infty}$-topology. A subset of either spaces is said to be generic if it is an open dense subset in the space.

The genericity of a property of germs are defined as follows. Let $P$ be a property of equation germs with complete integral $(\mu, f):\left(\mathbf{R}^{n+1}, 0\right) \rightarrow \mathbf{R} \times J^{1}\left(\mathbf{R}^{n}, \mathbf{R}\right)$ (respectively, Legendrian unfoldings $\left.\ell_{(\mu, f)}:\left(\mathbf{R}^{n+1}, 0\right) \rightarrow J^{1}\left(\mathbf{R} \times \mathbf{R}^{n}, \mathbf{R}\right)\right)$. For an open set $U \subset \mathbf{R}^{r}$, we define $\mathcal{P}(U)$ to be the set of $(\mu, f) \in \operatorname{Int}\left(U, \mathbf{R} \times J^{1}\left(\mathbf{R}^{n}, \mathbf{R}\right)\right.$ ) (respectively, $\ell_{(\mu, f)} \in$ $\left.L\left(U, J^{1}\left(\mathbf{R} \times \mathbf{R}^{n}, \mathbf{R}\right)\right)\right)$ such that the germ at $x$ whose representative is given by $(\mu, f)$ (respectively, $\ell_{(\mu, f)}$ ) has property $P$ for any $x \in U$.

The property $P$ is said to be generic if for some neighbourhood $U$ of 0 in $\mathbf{R}^{r}$, the set $\mathcal{P}(U)$ is a generic subset in $\operatorname{Int}\left(U, \mathbf{R} \times J^{1}\left(\mathbf{R}^{n}, \mathbf{R}\right)\right)\left(\right.$ respectively, $L\left(U, J^{1}\left(\mathbf{R} \times \mathbf{R}^{n}, \mathbf{R}\right)\right)$.

By the construction, we have a well-defined continuous mapping

$$
\left(\Pi_{1}\right)_{*}: L\left(U, J^{1}\left(\mathbf{R} \times \mathbf{R}^{n}, \mathbf{R}\right)\right) \rightarrow \operatorname{Int}\left(U, \mathbf{R} \times J^{1}\left(\mathbf{R}^{n}, \mathbf{R}\right)\right)
$$

defined by $\left(\Pi_{1}\right)_{*}\left(\ell_{(\mu, f)}\right)=\Pi_{1} \circ \ell_{(\mu, f)}=(\mu, f)$, where $\Pi_{1}: J^{1}\left(\mathbf{R} \times \mathbf{R}^{n}, \mathbf{R}\right) \rightarrow J^{1}\left(\mathbf{R}^{n}, \mathbf{R}\right)$ is the canonical projection. Then it has been shown that the following fundamental theorem.

\section{THEOREM 3.3. The continuous map}

$$
\left(\Pi_{1}\right)_{*}: L\left(U, J^{1}\left(\mathbf{R} \times \mathbf{R}^{n}, \mathbf{R}\right)\right) \rightarrow \operatorname{Int}\left(U, \mathbf{R} \times J^{1}\left(\mathbf{R}^{n}, \mathbf{R}\right)\right)
$$

is a homeomorphism.

Of course, the set of holonomic systems of Clairaut type is open by Porposition 3.2. We use the notion of generic properties of holonomic systems of Clairaut type in the set of all holonomic systems of Clairaut type adopting the relative topology as an open subset of $\operatorname{Int}\left(U, \mathbf{R} \times J^{1}\left(\mathbf{R}^{n}, \mathbf{R}\right)\right)$.

THEOREM 3.4. For a generic holonomic system of Clairaut type

$$
(\mu, f):\left(\mathbf{R}^{n+1}, 0\right) \rightarrow \mathbf{R} \times J^{1}\left(\mathbf{R}^{n}, \mathbf{R}\right),
$$


the integral diagram $(\mu, \pi \circ f)$ is strictly equivalent to one of germs in the following list: $D A_{1}$;

$$
\begin{aligned}
& \mu=u_{n+1}, \\
& g=\left(u_{1}, \ldots, u_{n+1}\right) .
\end{aligned}
$$

$D A_{2}$

$$
\begin{aligned}
& \mu=u_{n+1}-\frac{1}{2} u_{1} \\
& g=\left(u_{1}, \ldots, u_{n}, u_{n+1}^{2}\right)
\end{aligned}
$$

$D A_{\ell}(3 \leq \ell \leq n+1)$

$$
\begin{aligned}
& \mu=u_{n+1} \\
& g=\left(u_{1}, \ldots u_{n}, u_{n+1}^{\ell}+\sum_{i=1}^{\ell-1} u_{i} u_{n+1}^{i}\right)
\end{aligned}
$$

$\widetilde{D A}_{n+2}$

$$
\begin{aligned}
& \mu_{\alpha}=u_{n+1}+\alpha \circ g \quad \text { for } \alpha \in \mathfrak{M}_{(x, y)} \\
& g=\left(u_{1}, \ldots, u_{n}, u_{n+1}^{n+2}+\sum_{i=1}^{n} u_{i} u_{n+1}^{i}\right)
\end{aligned}
$$

This theorem gives a generic classification of integral diagrams of Clairaut type under the strict equivalence. However, our purpose is to classify these subjects by the equivalence. We remark that each germs of types $D A_{\ell}(2 \leq \ell \leq n+1)$ and $\widetilde{D A}_{n+2}$ are not equivalent. Thus the problem is reduced to classify germs which are contained in the family $\widetilde{D A}_{n+2}$ by the equivalence. This family is parametrized by function germs $\alpha$ which are called functional moduli. We shall characterize functional moduli relative to the equivalence. For the purpose, we now adopt coordinates $\left(x_{1}, \ldots, x_{n}, y\right)$ of $\mathbf{R}^{n} \times \mathbf{R}$ and define $\mathcal{D}^{n}=\left\{\left(x_{1}, \ldots, x_{n}, y\right) \in \mathbf{R}^{n} \times \mathbf{R} \mid F=\frac{\partial F}{\partial t}=\cdots=\frac{\partial^{n} F}{\partial t^{n}}=0\right.$ for some $\left.t\right\}$, where $F\left(t, x_{1}, \ldots, x_{n}, y\right)=t^{n+2}+x_{1} t+\cdots+x_{n} t^{n}-y$. Then we have the following characterization theorem.

THEOREM 3.5. Let $\left(\mu_{\alpha}, g\right)$ be an integral diagram of $\widetilde{D A}_{n+2}$. Then for any $\alpha$, there exists a function germ $\alpha^{\prime}:\left(\mathbf{R}^{n+1}, 0\right) \rightarrow(\mathbf{R}, 0)$ such that

(1) $\left(\mu_{\alpha}, g\right)$ is equivalent to $\left(\mu_{\alpha^{\prime}}, g\right)$.

(2) $\alpha^{\prime} \mid \mathcal{D}^{n}=0$.

This theorem is a generalization of Dufour's result in [2]. He has also shown that the uniqueness of functional moduli relative to the equivalence, so that we now consider a generalization of his uniqueness result. Define

$$
\begin{gathered}
\Delta=\left\{\left(x_{1}, \ldots, x_{n}, y\right) \in \mathbf{R}^{n} \times \mathbf{R} \mid \text { The }(n+2)\right. \text {-degree algebraic equation } \\
\left.F_{\left(x_{1}, \ldots, x_{n}, y\right)}(t)=0 \text { has }(n+2) \text {-real roots with multiplicity }\right\},
\end{gathered}
$$

where $F_{\left(x_{1}, \ldots, x_{n}, y\right)}(t)=F\left(t, x_{1}, \ldots, x_{n}, y\right)$. We say that $\alpha$ and $\alpha^{\prime}$ are equivalent as moduli if there exists $a \in \mathbf{R}-\{0\}$ such that $a \alpha\left(x_{1}, \ldots, x_{n}, y\right)=\alpha^{\prime}\left(a^{n+1} x_{1}, a^{n} x_{2}, \ldots, a^{2} x_{n}\right.$, $\left.a^{n+2} y\right)$ for any $\left(x_{1}, \ldots, x_{n}, y\right) \in \Delta$. We remark that this definition of the equivalence 
among functional moduli is slightly different from Dufour's definition of it in [2]. If we adopt his definition, we cannot assert the necessity of the condition that functional moduli are equivalent. Then we correct the definition as the above.

THEOREM 3.6. Let $\left(\mu_{\alpha}, g\right)$ and $\left(\mu_{\alpha^{\prime}}, g\right)$ be integral diagrams of $\widetilde{D A}_{n+2}$ such that $\alpha\left|\mathcal{D}^{n}=\alpha^{\prime}\right| \mathcal{D}^{n}=0$. Then $\left(\mu_{\alpha}, g\right)$ and $\left(\mu_{\alpha^{\prime}}, g\right)$ are equivalent if and only if $\alpha$ and $\alpha^{\prime}$ are equivalent as moduli.

We emphasize that this theorem asserts that the equivalence classes of functional moduli $\alpha$ with $\alpha \mid \mathcal{D}^{n}=0$ are the complete invariants for generic classifications of holonomic systems of Clairaut type under the equivalence relation given by the group of point transformations.

We define $\mathfrak{M}\left(\mathcal{D}^{n}\right)=\left\{\alpha \in \mathfrak{M}_{(x, y)}|\alpha| \mathcal{D}^{n}=0\right\}$ and $\mathcal{M}\left(\widetilde{D A}_{n+2}\right)=\mathfrak{M}\left(\mathcal{D}^{n}\right) / \sim$, where $\sim$ denotes the equivalence relation as moduli. The above theorem asserts that the moduli space for generic holonomic systems of Clairaut type is $\mathcal{M}\left(\widetilde{D A}_{n+2}\right)$.

\title{
REFERENCE
}

[1] A.C. Clairaut, Solution de plusieurs problems, Histore de l'Academie royale de Sciences, Paris (1734), 196-215.

[2] J.-P. Dufour, Modules pour les familles de courbes planes, Annales de l'Institut Fourier (1989), 225-238.

[3] S. Izumiya, Completely integrable holonomic systems of first order differential equations, to appear in Proceedings of the Royal Society of Edinburgh.

[4] S. Izumiya, The theory of Legendrian unfoldings and first order differential equaitions, Proceedings of the Royal Society of Edinburgh (1993), 123A, 517-532.

\author{
Department of Mathematics \\ FACULTY OF SCIENCE \\ HOKKAIDO UNIVERSITY \\ SAPPORO 060, JAPAN \\ e-mail address: \\ izumiya@math.hokudai.ac.jp \\ AND \\ y-kurokw@math.hokudai.ac.jp
}

